\title{
Prevalence of Ventricular Ectopy in Older Adults across Different Frailty Levels
}

\author{
Saman Parvaneh ${ }^{1,2,3}$, Bijan Najafi ${ }^{1,2,5}$, Nima Toosizadeh ${ }^{2}$, Irbaz Bin Riaz ${ }^{4}$, Jane Mohler ${ }^{1,2}$ \\ ${ }^{1}$ University of Arizona, Department of Surgery, Tucson, USA \\ ${ }^{2}$ University of Arizona, Department of Medicine, Arizona Center on Aging, Tucson, USA \\ ${ }^{3}$ Philips Research North America, Cambridge, USA \\ ${ }^{4}$ University of Arizona, Department of Internal Medicine, Tucson, USA \\ ${ }^{5}$ Baylor College of Medicine, Department of Surgery, Houston, USA
}

\begin{abstract}
An increased prevalence of ventricular ectopy (Premature Ventricular Contraction, PVC) is associated with increased incidence of congestive heart failure and increased mortality. Increase in incidence of ventricular ectopy with age has been demonstrated in previous studies, but to the best of our knowledge, its association with frailty, a geriatric syndrome that is associated with adverse health outcomes, has not been studied. The aim of this study was to assess the prevalence of ventricular ectopy in older adults across different frailty levels. A unichannel ECG in 45 older adults aged 65 and above were measured using a wearable ECG recorder. Participants were classified as non-frail $(n=22)$, pre-frail $(n=17)$, and frail $(n=6)$ using the Fried frailty phenotype. PVCs were identified automatically, and subsequently checked manually by an expert to ensure accuracy. Number of PVC beats per hour was considered for ECG assessment and analysis of variance (ANOVA) test was used to evaluate the number of PVC episodes per hour among frailty groups. Ninety eight percent of participants had at least one PVC episode and correlation of number of PVC beats with age was very weak $(r=0.28, p=0.076)$. Number of $P V C$ beats was not significantly different $(p>0.05)$

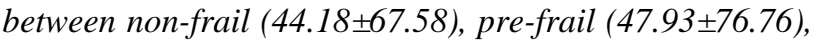
and frail (22.13 \pm 7.84$)$. However, the average number of $P V C$ beats was higher in pre-frail compared to non-frail $(p>0.05)$.
\end{abstract}

\section{Introduction}

Frailty as a geriatric syndrome is associated with increased risk of adverse health events such as falls, hospitalization, morbidity, and mortality [1, 2]. Frail people are more vulnerable to acute and chronic conditions such as cardiac surgery and heart disease (e.g., myocardial infarction and heart failure) [3, 4]. Also, frailty is higher in older adults with cardiovascular disease $[3,5]$.
Assessment of heart rate variability confirmed the impairment of cardiac autonomic nervous system in frail compared to non-frail participants [5, 6]. Although, previous studies demonstrated that incidence of ventricular ectopy (Premature Ventricular Contraction, PVC) increases with age [7], but to the best of our knowledge its association with frailty, a geriatric syndrome that is associated with adverse health outcomes, has not been studied. An increased prevalence of ventricular ectopy is associated with increased incidence of congestive heart failure (CHF) and increased mortality [8]. Therefore, the aim of this study was to study the prevalence of PVC in older adults across different frailty categories.

\section{Method and material}

\subsection{Participants}

Forty five female older adults aged 65 and above (age: $76.12 \pm 7.82$ years, body mass index (BMI): $28.71 \pm 6.68$ $\left.\mathrm{kg} / \mathrm{m}^{2}\right)$ ) without diagnosed cardiovascular disease were recruited from geriatric clinics in a southwestern tertiary academic medical center after completing written informed consent. The study was approved by the University of Arizona Institutional Review Board.

\subsection{Frailty assessment}

Participants were classified as non-frail, pre-frail, and frail using the validated Fried frailty phenotype [2]. Frailty criteria included unintentional weight loss, slowness (walking speed for 4.6 meter distance), weakness (handgrip strength), and self-reported low physical activity and exhaustion. Participants with three or more positive frailty criteria were considered frail, individuals with one or two positive frailty criteria were considered pre-frail, and those with none of above criteria classified as nonfrail. 


\subsection{ECG monitoring and analysis}

A commercial FDA approved body-worn ECG recorder, BioHarness3 bio-patch (Zephyr Technology, Annapolis, MD), was used to record four hours of unichannel ECG in participants. The bio-patch was attached to the skin using standard ECG electrodes and ECG was sampled at frequency $250 \mathrm{~Hz}$. A code developed in Matlab (Mathworks, Natick, MA) was employed to automatically identify PVCs, and subsequently checked manually by an expert to ensure accuracy.

For automatic detection of PVC, presence of a compensatory pause after PVC as a significant characteristic of PVC, was captured using RR intervals [9]. Of note, QRS peaks were detected using Pan-Tompkins algorithm and were used for estimation of RR intervals [10]. Number of PVC beats per hour (\#PVC/hour) was considered for ECG assessment.

\subsection{Statistical analysis}

The association between PVC occurrence and age was assessed using Pearson's correlation. Correlation range of $0.01-0.19,0.20-0.39,0.40-0.59,0.60-0.79$, and $0.80-1.00$ were considered as very weak, weak, moderate, strong, and very strong association, respectively [11].

Analysis of variance (ANOVA) with Least Significant Difference (LSD) post-hoc analysis or chi-square $\left(\chi^{2}\right)$ test were used to evaluate differences in demographics, health parameters, and extracted parameter among frailty groups. Statistical level of significance was set to $p=0.05$.

Furthermore, coefficient of variation (CV), defined as ratio of the standard deviation to the mean, was used as a measure of the variability in extracted parameter [12].

\section{Results and discussion}

Forty-four participants (97\%) had at least one PVC episode. In previous studies in different aging groups (e.g., young, middle-age, and elderly), $40 \%-100 \%$ of participants had at least one PVC episode [13, 14]. This number increases with age to more than 70\% [13] and PVC burden increases 34\% for each 5-year increment increase in age [14].

Number of PVC beats per hour in studied population was $42.65 \pm 66.32$ and had a weak correlation with age $(r=0.28, p=0.076)$. Number of PVC per hour in this study is similar to previous work that demonstrate that this number was less than 100 [13].

Using Fried's frailty criteria, 22 participants were classified as non-frail (49\%), 17 were categorized as prefrail (38\%), and 6 were classified as frail (13\%). Demographic and clinical characteristics of participants are listed in Table 1. Between health information, only BMI was significantly different between non-frail and frail groups ( $p=0.004)$. Among number/percentage of observed frailty criteria, weakness and exhaustion were more prevalent in frail group compared to pre-frail group $(p<0.05)$.

Number of PVC beats per hour was not significantly different ( $>0.05$ ) between non-frail (44.18 \pm 67.58$)$, prefrail (47.93 \pm 76.76$)$, and frail $(22.13 \pm 7.84)$. This results was in agreement with Chao et al.'s observation which demonstrated no significant difference between severity of frailty assessed by self-report frailty instruments between patients with, and without, PVCs [15].

Interestingly, the average number of PVC beats was, respectively, $52 \%$ and $54 \%$ less in frail population compared to non-frail and pre-frail. Although, no significant association between prevalence of PVC and frailty level was found in this study, but the average number of PVC beats was 8\% higher in pre-frail compared to non-frail. Therefore, higher occurrence of PVCs may be an indicator of pre-frailty.

The higher variation in occurrence of PVCs for non-frail $(\mathrm{CV}=1.53)$ and pre-frail $(\mathrm{CV}=1.60)$ compared to frail $(\mathrm{CV}=0.35)$ may be related to the diversity of participants in these two groups, but possible reason(s) of higher variability needs to be explored in future studies.

Table 1. Demographic and health information of participant expressed in mean \pm standard deviation across non-frail, pre-frail, and frail groups and number/percentage of observed frailty criteria $(*: p<0.05)$.

\begin{tabular}{lcccc}
\hline Characteristic & Non-frail & Pre-frail & Frail & $p$-value \\
\hline Participants, $\mathrm{n}$ & 22 & 17 & 6 & - \\
Age, years & $74.36 \pm 6.41$ & $76.91 \pm 7.86$ & $80.33 \pm 11.43$ & 0.224 \\
Height, cm & $160.22 \pm 5.86$ & $157.73 \pm 6.77$ & $159.91 \pm 8.50$ & 0.490 \\
BMI, kg/m & $26.28 \pm 5.28$ & $29.69 \pm 7.49$ & $34.85 \pm 4.64$ & $0.012^{*}$ \\
\hline \multicolumn{2}{c}{ Observed frailty criteria, $\mathrm{n}$} & & & \\
Weight Loss & - & $1(5.9 \%)$ & $0(0 \%)$ & 0.544 \\
Slowness & - & $10(59 \%)$ & $6(100 \%)$ & 0.059 \\
Weakness & - & $6(35 \%)$ & $5(83 \%)$ & $0.043^{*}$ \\
Low Activity & - & $2(1.2 \%)$ & $3(50 \%)$ & $0.051^{*}$ \\
Exhaustion & - & $2(1.2 \%)$ & $5(83 \%)$ & $0.001^{*}$ \\
\hline
\end{tabular}

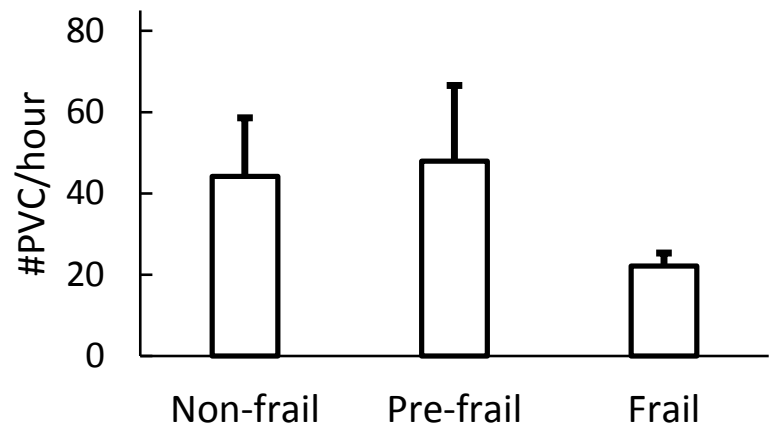

Figure 1. Number of PVC episodes per hour across groups with different frailty status. 
When compared between groups with and without different frailty criteria (i.e., slowness, weakness, low activity, and exhaustion), no significant between-group differences were observed for number of PVC episodes per hour (Table 2). Since weight loss was present in only one participant (2\%), this criteria was not included in Table 2. In this study, only female participants were included to reduce the effect of gender as a confounding parameter. Also, the number of frail participants (13\%) was small. Therefore, another study with a larger sample size including, both male and female participants, is warranted to evaluate the association of PVC prevalence and frailty status.

Table 2. Number of PVC episodes per hour (mean \pm standard) between groups with and without different frailty criteria $(*: p<0.05)$.

\begin{tabular}{lccc}
\hline Characteristic & With & Without & $\begin{array}{c}p- \\
\text { value }\end{array}$ \\
\hline Slowness & $33.47 \pm 38.43$ & $47.72 \pm 77.76$ & 0.496 \\
Weakness & $16.18 \pm 8.72$ & $51.22 \pm 74.38$ & 0.129 \\
Low Activity & $26.70 \pm 16.91$ & $44.65 \pm 69.98$ & 0.574 \\
Exhaustion & $40.96 \pm 54.33$ & $42.97 \pm 68.93$ & 0.942 \\
\hline
\end{tabular}

\section{Conclusions}

We report the association of ventricular ectopy occurrence (number of PVC beats per hour) and frailty in this article. In a cross-sectional ECG analysis, the average number of PVC beats was higher in the pre-frail group compared to the non-frail group. Therefore, future longitudinal studies are recommended to explore the association between frailty status and different heart arrhythmia over the time.

\section{Acknowledgements}

This study was partly supported by an STTR-Phase II Grant (award No. 2R42AG032748) from the National Institute on Aging and the Arizona Center on Aging's Hudson family. The content is solely the responsibility of the authors and does not necessarily represent the official views of the National Institute on Aging or the National Institutes of Health. We thank Marilyn Gilbert and the coordination team for helping with data collection and also Dr. Hossein Mehdikhani for assisting in manual review of PVC episodes.

\section{References}

[1] L. Ferrucci, C. Cavazzini, A. Corsi, B. Bartali, C. Russo, F. Lauretani, et al., "Biomarkers of frailty in older persons," Journal of endocrinological investigation, vol. 25, pp. 1015, 2001.

[2] L. P. Fried, C. M. Tangen, J. Walston, A. B. Newman, C.
Hirsch, J. Gottdiener, et al., "Frailty in older adults evidence for a phenotype," The Journals of Gerontology Series A: Biological Sciences and Medical Sciences, vol. 56, pp. M146-M157, 2001.

[3] J. Afilalo, K. P. Alexander, M. J. Mack, M. S. Maurer, P. Green, L. A. Allen, et al., "Frailty assessment in the cardiovascular care of older adults," Journal of the American College of Cardiology, vol. 63, pp. 747-762, 2014.

[4] K. M. Flint, D. D. Matlock, J. Lindenfeld, and L. A. Allen, "Frailty and the selection of patients for destination therapy left ventricular assist device," Circulation: Heart Failure, vol. 5, pp. 286-293, 2012.

[5] S. Parvaneh, C. L. Howe, N. Toosizadeh, B. Honarvar, M. J. Slepian, M. Fain, et al., "Regulation of cardiac autonomic nervous system control across frailty statuses: a systematic review," Gerontology, vol. 62, pp. 3-15, 2015.

[6] S. Parvaneh, N. Bijan, and J. Mohler, "Study of Cardiac Autonomic Nervous System in Older Adults with Different Frailty Status," in Gerontology Society of America (GSA), 2014.

[7] H. Tasaki, T. Serita, C. Ueyama, K. Kitano, S. Seto, K. Yano, et al., "Longitudinal age-related changes in 24-hour total heart beats and premature beats and their relationship in healthy elderly subjects," International heart journal, vol. 47, pp. 549-563, 2006.

[8] J. W. Dukes, T. A. Dewland, E. Vittinghoff, M. C. Mandyam, S. R. Heckbert, D. S. Siscovick, et al., "Ventricular ectopy as a predictor of heart failure and death," Journal of the American College of Cardiology, vol. 66, pp. 101-109, 2015.

[9] M. G. Tsipouras, D. I. Fotiadis, and D. Sideris, "An arrhythmia classification system based on the RR-interval signal," Artificial intelligence in medicine, vol. 33, pp. 237 250, 2005.

[10] J. Pan and W. J. Tompkins, "A real-time QRS detection algorithm," IEEE transactions on biomedical engineering, pp. 230-236, 1985.

[11] I. Lawrence and K. Lin, "A concordance correlation coefficient to evaluate reproducibility," Biometrics, pp. 255-268, 1989.

[12] B. Everitt, Cambridge dictionary of statistics: Cambridge University Press, 1998.

[13] K. A. Eagle and R. R. Baliga, Practical cardiology: evaluation and treatment of common cardiovascular disorders: Lippincott Williams \& Wilkins, 2008.

[14] J. Luebbert, D. Auberson, and F. Marchlinski, "Premature Ventricular Complexes in Apparently Normal Hearts," Cardiac Electrophysiology Clinics, vol. 8, pp. 503-514, 2016.

[15] C.-T. Chao and J.-W. Huang, "Frailty severity is significantly associated with electrocardiographic QRS duration in chronic dialysis patients," PeerJ, vol. 3, p. e1354, 2015.

Address for correspondence.

Jane Mohler

Arizona Center on Aging, University of Arizona

1807 Elm Street, Tucson, AZ 85724

jmohler@aging.arizona.edu 
